\title{
Cooperative Spectrum Sensing Optimization Algorithm Based on Adaptive Threshold Setting
}

\author{
Tangsen Huang ${ }^{1}$ and Jie-tai Wang ${ }^{2 *}$ \\ ${ }^{1}$ School of electronics and Information Engineering, \\ Hunan University of science and Engineering, Yongzhou, 425199, China \\ ${ }^{2 *}$ College of Mathematics Physics and Information Engineering, \\ Jiaxing University, Jiaxing 314001, China \\ ${ }^{1}$ huangtangsen@163.com, ${ }^{2 *}$ taiiwang@163.com
}

\begin{abstract}
Energy detection performance in the signal to noise ratio (SNR) fluctuation needs to be improved. In order to obtain the highest probability of detection, a new cooperative spectrum sensing algorithm is proposed. Dynamic double energy thresholds and adaptive grid search are utilized to obtain the highest probability detection. Double thresholds are adaptive to noise fluctuation, and in order to obtain the best sensing performance, adaptive grid search is used firstly to find the optimal double thresholds. Simulation results show that the proposed algorithm has excellent robustness to noise fluctuation and good sensing performance even under the low signal to noise ratio.
\end{abstract}

Keywords: Cognitive radio, spectrum sensing, double thresholds, grid search

\section{Introduction}

In cognitive radio networks, second user (SU) can utilize a licensed channel when the signal of the primary user (PU) is absent [1]. In order to assess whether the primary user is present or absent. SU must perform spectrum sensing [2]. The initial sensing method is single SU performance spectrum sensing independently. Due to path loss, Rayleigh fading and shadowing fading, single SU performance spectrum sensing poorly [3]. So as to obtain better sensing performance. Some SU can jointly perform cooperative spectrum sensing [4]. In this paper, considering the path loss, each SU collects different energy and need in order to adjust double energy thresholds for enhancing reliability of local decision. Therefore, a control parameter to accurately fine tune the double thresholds is introduced. Further, in order to reflect the difference of SU collected energy, each SU will be assigned a weight according to its collected energy. Fusion center will fuse their weights according to majority rule and make a final decision to determine the channel is free or busy. Simulation results show that the proposed sensing scheme has excellent performance and outperforms the conventional sensing schemes under low SNR.

Some studies are focusing on cooperative sensing using double energy threshold. In [5], hierarchical cooperative sensing method was used to improve sensing performance. The distance between double thresholds was divided into four regions and all weights in a region are same. But this method was not taken the particularity of each SU into serious consideration. In [6], eigenvalue of the signal was used to improve sensing performance, but the distance between double thresholds was subjectively given and the double energy thresholds in this method were not robust to noise fluctuation. In [7], k-out-of-M fusion rule was used to improve sensing performance. In this method, the difference between double thresholds was important to optimize the detection performance, but the authors did not explain how to set the double thresholds appropriately.

In this paper, a new method of estimate noise power and SNR is proposed. The proposed double thresholds have capability to adapt to the change of noise power. Due to 
the different SU estimates noise power and SNR, all SU perform local sensing will get different double thresholds, and sensing results are more reliable compared with all SU have the same double thresholds. Energy of each SU received is different, all SU are respectively allocated weights according to their receiving energy, then participated in cooperative sensing. The proposed sensing method, cooperative sensing uses voting rule so that each SU can make the appropriate sensing contribution. Due to the proposed double thresholds is dynamic, it is necessary to solve how to set the double thresholds. In this paper, we set an impact factor to adjust the distance between the double thresholds. When the impact factor has been changed, double thresholds and sensing performance will be changed. Therefore, it is important to find out the optimal impact factor to improve sensing performance. Grid search can find out the optimal impact factor [8]. In order to improve search efficiency, we are the first one using adaptive grid search to find out the optimal impact factor and obtain the best sensing performance. Simulation results show that the proposed cooperative sensing method achieves better sensing performance even under low SNR and noise fluctuation.

\section{System Model}

In the following section, a system having $N$ SUs is considered, a PU and a cognitive base station. All SUs communicate with the cognitive base station through a common control channel, and the cognitive base station is a fuse center and it decides PU is present or absent.

\subsection{Calculate the Optimal Threshold}

For spectrum sensing, every SU performs energy detection independently and the received signal of $\mathrm{SU}$ is given by

$$
x(m)=s(m)+v(m), m=0,1, \cdots, \mathrm{M}-1
$$

where $s(m)$ is transmitted signal of PU, $v(m)$ is additive white Gaussian noise (AWGN) with zero mean and variance of $\sigma_{v}{ }^{2}$, and $\mathrm{M}$ is the number of samples. PU absence is denoted by $H_{0}$, and PU presence is denoted by $H_{1}$. So they can be expressed as [5]

$$
\begin{array}{ll}
H_{0}: x(m)=v(m), & m=0,1, \ldots, M-1 \\
H_{1}: x(m)=s(m)+v(m), & m=0,1, \ldots, M-1
\end{array}
$$

Assume $\mathrm{E}$ is the average energy of the $\mathrm{SU}$ received signal, it is expressed as [6]

$$
E=\frac{1}{M} \sum_{m=0}^{M-1}|x(m)|^{2}
$$

When $\mathrm{M}>250$, E follows Gaussian distribution according to the central limitation theorem. If each SU makes its local decision depend on a single threshold $\lambda_{0}$, then the probability of detection $\left(P_{d}\right)$ and the probability of false alarm $\left(P_{f}\right)$ can be expressed as respectively

$$
\begin{gathered}
P_{d}=P\left\{E \geq \lambda_{0} \mid H_{1}\right\}=Q\left(\frac{\lambda_{0}-\left(\sigma_{s}{ }^{2}+\sigma_{v}{ }^{2}\right)}{\left(\sigma_{s}{ }^{2}+\sigma_{v}{ }^{2}\right) / \sqrt{M / 2}}\right) \\
P_{f}=P\left\{E \geq \lambda_{0} \mid H_{0}\right\}=Q\left(\frac{\lambda_{0}-\sigma_{v}{ }^{2}}{\sigma_{v}{ }^{2} / \sqrt{M / 2}}\right)
\end{gathered}
$$

where $\sigma_{s}^{2}$ is signal power of PU, $\sigma_{v}^{2}$ is noise power, $\lambda_{0}$ is decision threshold, and $Q(x)$ is expressed as [7]

$$
Q(x)=\frac{1}{\sqrt{2 \pi}} \int_{x}^{\infty} e^{\frac{-t^{2}}{2}} d t
$$


In order to improve $P_{d}$ and reduce $P_{f}$ simultaneously, we put a limiting range on the threshold $\lambda_{0}$ as

$$
\sigma_{v}^{2} \leq \lambda_{0} \leq \sigma_{v}{ }^{2}+\sigma_{s}^{2}
$$

If the probability of PU presence is $P\left(H_{1}\right)=\beta, 0<\beta<1$ the probability of $\mathrm{PU}$ absent is $P\left(H_{0}\right)=1-\beta$ and the probability of error detection $\left(P_{e}\right)$ is expressed as [8] $P_{e}=P\left(H_{1}\right)\left(1-P_{d}\right)+P\left(H_{0}\right) P_{f}=\beta\left(1-P_{d}\right)+(1-\beta) P_{f}$

Because $P_{d}, P_{f}$ and $P_{e}$ are quadratic function of the $\lambda_{0}$, we can derive . There must have the optimal threshold $\lambda_{0}$ to minimize $P_{e}$, and then by letting $\frac{\partial P_{e}}{\partial \lambda_{0}}=0$ to obtain the optimal threshold $\lambda_{o}$, it is expressed as

$$
\lambda_{o}=\left|\frac{\sigma_{v}^{2}(1+\gamma)\left[1+\sqrt{1+\frac{4(2+\gamma)}{M \cdot \gamma} \ln \left[\frac{(1-\beta)(1+\gamma)}{\beta}\right]}\right.}{2+\gamma}\right| \mid
$$

where $\gamma=\frac{\sigma_{s}^{2}}{\sigma_{v}{ }^{2}}$ is the SNR of the receiving terminal of SU. If we know the signal power $\sigma_{s}^{2}$, noise power $\sigma_{v}^{2}$ and the SNR of the receiving terminal, it is easy to obtain the optimal threshold. If collecting the energy in a sensing cycle is $E$, and $E$ can be divided into two equal sections $E_{1}$ and $E_{2}$.

If $E_{1}<E_{2}$, we let $E_{1}=\hat{\sigma}_{v}^{2}, E_{2}=\hat{\sigma}_{s}^{2}+\hat{\sigma}_{v}^{2}$ and the estimated value of SNR is

$$
\hat{\gamma}=\frac{\hat{\sigma}_{s}^{2}}{\hat{\sigma}_{v}^{2}}=\frac{E_{2}-E_{1}}{E_{1}}
$$

Then the optimal threshold $\lambda_{o}$ can be estimated by the following expression

$$
\hat{\lambda}_{o}=\left|\frac{\hat{\sigma}_{v}^{2}(1+\hat{\gamma})\left[1+\sqrt{1+\frac{4(2+\hat{\gamma})}{(M / 2) \hat{\gamma}} \ln \left[\frac{(1-\beta)(1+\hat{\gamma})}{\beta}\right]}\right.}{2+\hat{\gamma}}\right| \mid
$$

\subsection{Adaptive Double-threshold Setting}

The optimal threshold $\hat{\lambda}_{O}$ is the center point, and $d(d \geq 0)$ is distance between $\hat{\lambda}_{o}$ and threshold $\lambda_{h}$ larger than $\hat{\lambda}_{o}$ or threshold $\lambda_{l}$ lower than $\hat{\lambda}_{o}$.

In fact, noise power should be less than or equal to $\lambda_{l}$, and noise power adding signal power should be greater than or equal to $\lambda_{h}$, so $d$ should satisfy

$$
\begin{gathered}
E_{1}=\hat{\sigma}_{v}^{2} \leq \hat{\lambda}_{o}-d=\lambda_{l} \\
\lambda_{h}=\hat{\lambda}_{o}+d \leq \hat{\sigma}_{v}^{2}+\hat{\sigma}_{s}^{2}=E_{2}
\end{gathered}
$$


$(12)+(13)$ can obtain

$$
0 \leq d \leq \frac{1}{2}\left(E_{2}-E_{1}\right)
$$

Let

$$
d=\varepsilon\left(E_{2}-E_{1}\right)
$$

where $\varepsilon$ is an impact factor, and $0 \leq \varepsilon \leq 0.5$. When $\varepsilon=0$, double thresholds problem is changed to single threshold problem. $\lambda_{h}$ and $\lambda_{l}$ can be expressed as

$$
\begin{aligned}
& \lambda_{h}=\hat{\lambda}_{o}+\varepsilon\left(E_{2}-E_{1}\right) \\
& \lambda_{l}=\hat{\lambda}_{o}-\varepsilon\left(E_{2}-E_{1}\right)
\end{aligned}
$$

\section{Cooperative Spectrum Sensing Algorithm}

$N$ SUs take part in cooperative spectrum sensing in cognitive radio networks. Assume the $i^{t h} \mathrm{SU}$ receives the average energy of signal is $E_{i}, \lambda_{h, i}, \lambda_{l, i}, \hat{\lambda}_{o, i}$ and $w_{i}$ are the larger threshold, lower threshold, the estimated value of the optimal threshold and weight of $i^{\text {th }} \mathrm{SU}$, respectively.

All SUs are not the same distance with the PU, because path loss, multipath Rayleigh fading. Each SU receives different average energy of signal. That is to say, each SU maybe has different weight. The proposed method considers different SUs have different weights and roles in cognitive radio networks, this is close to the actual situation. All SUs contribute to the cooperative sensing according to their weights by fusion center. Algorithm 1 summarizes the weights allocation and cooperative sensing.

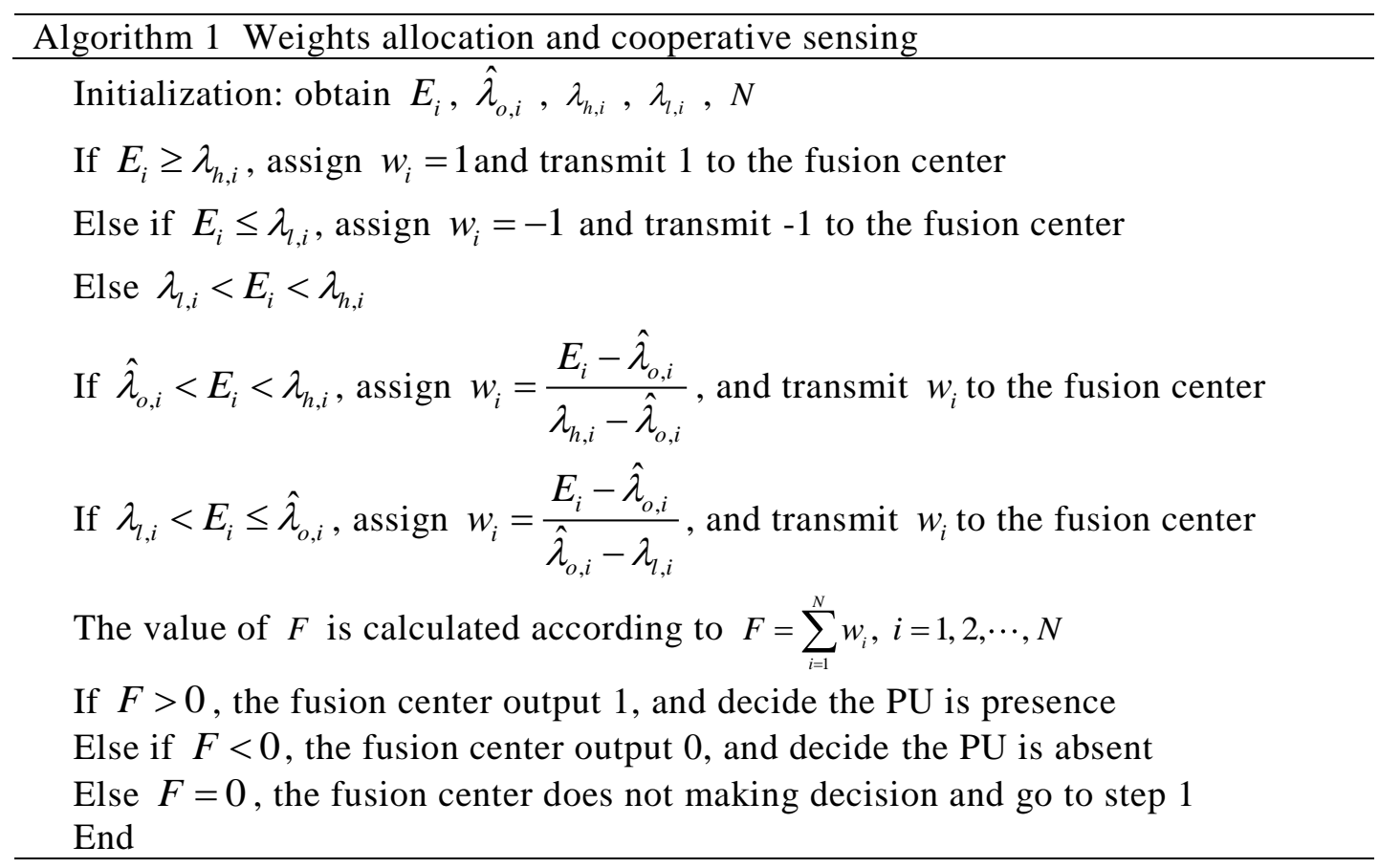

From algorithm 1, we discover that the fusion of all SUs are combined soft fusion with hard fusion, so algorithm 1 can improve sensing performance while reducing communication overhead. 


\section{Adaptive Grid Search Algorithm}

Setting of appropriate double thresholds will affect the sensing performance. The double thresholds are related to impact factor $\varepsilon$, that is to say which $\varepsilon$ can impact the sensing performance. When $0 \leq \varepsilon \leq 0.5$, there must exist a $\varepsilon$ making the best sensing performance. So it is important to ensure the optimal $\varepsilon$ to obtain the best sensing performance. Adaptive grid search can find out it to obtain the best sensing performance. Algorithm 2 explains the process using the adaptive grid search to find the optimal $\varepsilon$ and obtain the highest $P_{d}$.

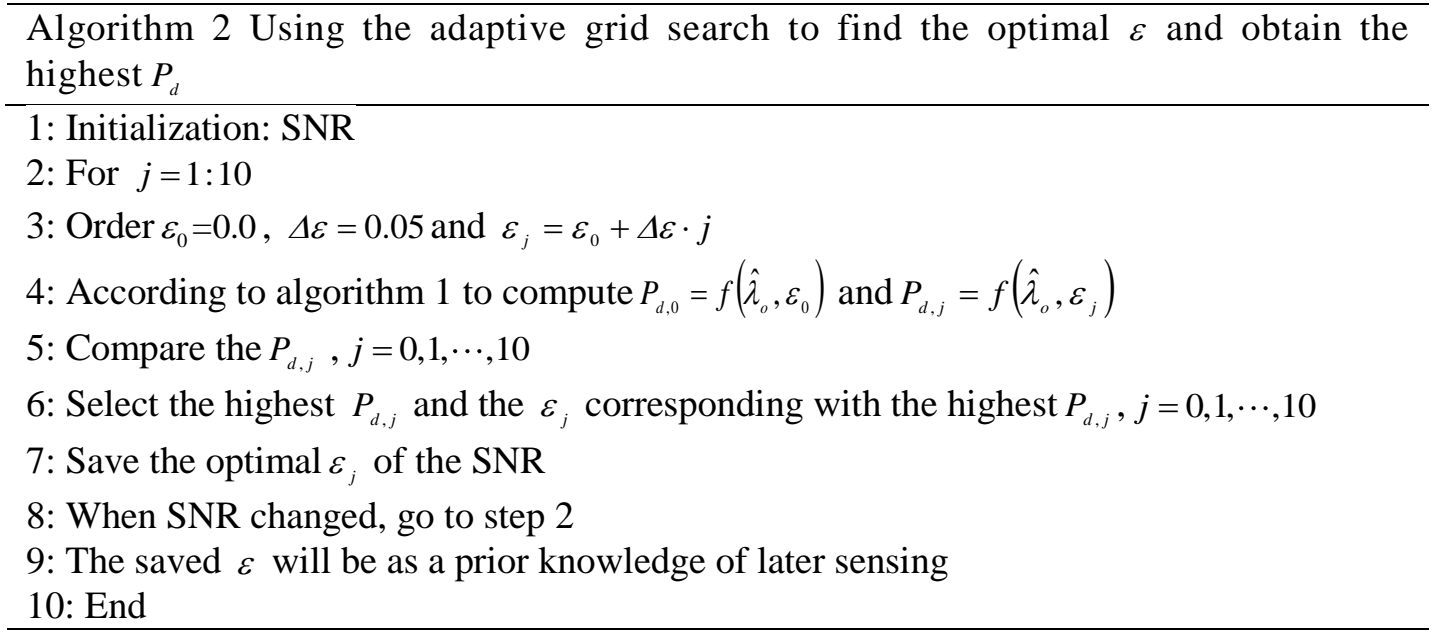

\section{Simulation Results}

For evaluating the sensing performance of the proposed sensing scheme, a lot of simulation results are shown. The signal of the PU is BPSK (Binary Phase Shift Keying) signal, the bandwidth is $10 \mathrm{KHz}$, the sensing time is $0.1 \mathrm{~s}$. Assume the $P\left(H_{1}\right)=\beta=0.5$ and the transmission power of the $\mathrm{PU}$ is $0.1 \mathrm{~W}$. We consider a $1 \mathrm{Km} \times 1 \mathrm{Km}$ area, PU located at the center, there are 16 SUs uniformly distributed in the area, Figure 1 illustrates the distribution of PU and SUs, and consider the impact of path loss, multipath Rayleigh fading and shadows shade, assume the path loss exponent is 3 , the standard deviation of the shadow is $6 \mathrm{~dB}$ and the mean of the multipath Rayleigh fading is 1 [15].

Figure 2 illustrates the results of the $P_{d}$ versus $\varepsilon, \varepsilon$ value is changed. Can be seen that when SNR is fixed, the $P_{d}$ changes along with $\varepsilon$ value changed from Figure 2, but there is an optimal $\varepsilon$ value to maximize the $P_{d}$. And different SNR have a different optimal $\varepsilon$ value. Due to SNR is often changing, need to use adaptive grid search algorithm to find out the optimal values. 


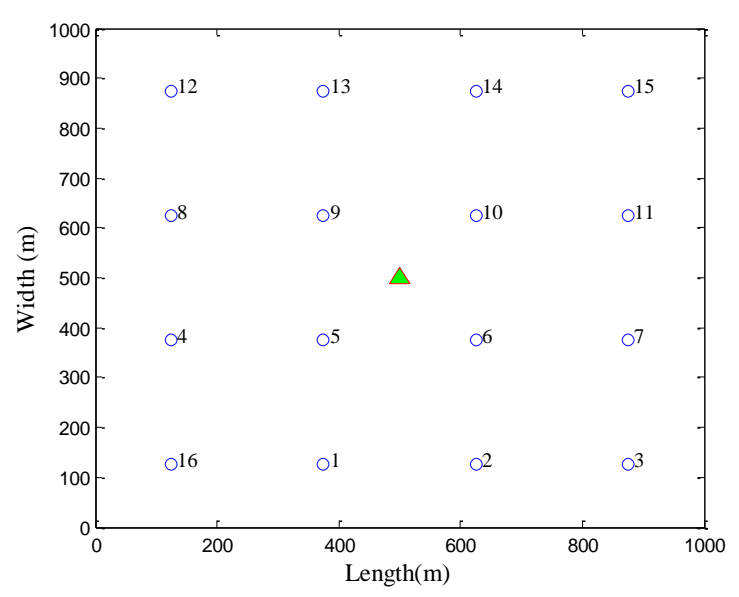

Figure 1. The Network used for Simulation, the Triangle Denote the PU, the Circles Denotes the SUs

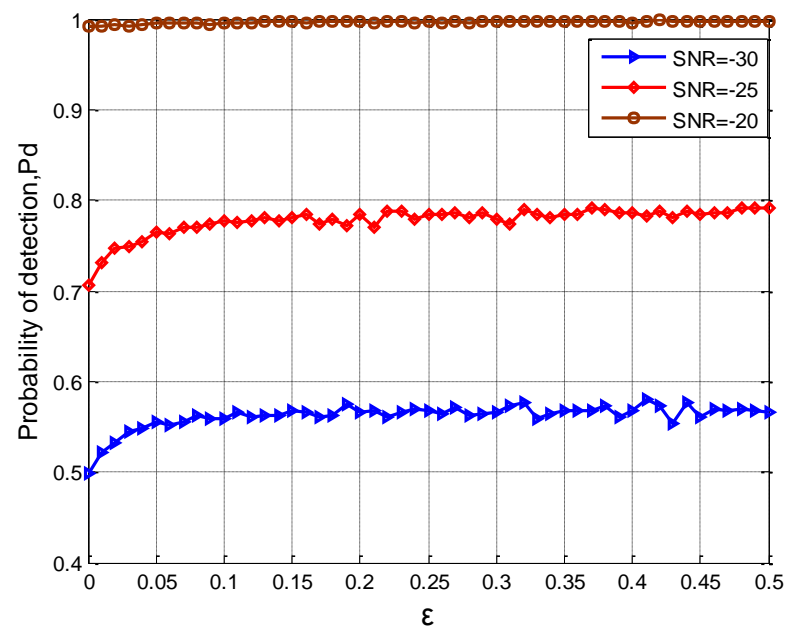

Figure 2. $P_{d}$ versus $\varepsilon$ for Cooperative Spectrum Sensing, $0 \leq \varepsilon \leq 0.5, \Delta \varepsilon=0.01$, SNR=30 dB, $-25 \mathrm{~dB}$ and $\mathbf{- 2 0 \mathrm { dB }}$

Figure 3 illustrates the results of using adaptive grid search algorithm to find out the optimal $\varepsilon$ values to maximize the $P_{d}$ in different SNR. Table 1 show the data consistent with the characteristic curve in Figure 3. Table 1 illustrates the results of using grid search to find the optimal $\varepsilon$ and obtain the highest $P_{d}$ under different SNR, and compared with fixed $\varepsilon$. Using grid search to obtain the probability of detection is higher than this of fixed $\varepsilon$. It also shows that using grid search has obvious effect to improve probability of detection, and has excellent robustness to noise fluctuation.

Figure 4 illustrates the results of the proposed spectrum sensing method compare with others sensing methods. There are 5 kinds of comparison methods, respectively are hierarchical with quantization method [6], double thresholds detection using eigenvalue method [7], the proposed adaptive single threshold method, the proposed adaptive double threshold method and the proposed adaptive grid search algorithm. Can be seen that the $P_{d}$ of obtain using adaptive grid search algorithm is highest from Figure 4, even using the proposed single threshold method, the $P_{d}$ outperforms others sensing methods. 


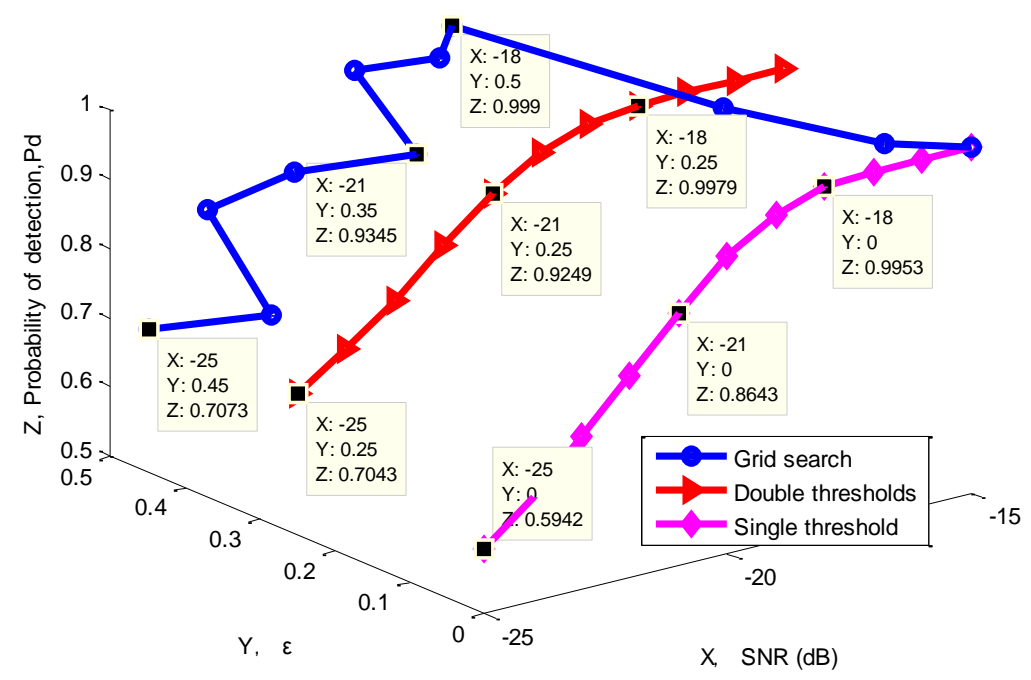

Figure 3. Comparison of $P_{d, \max }, P_{d, d}$ and $P_{d, s}$

Table 1. The Probability of Detection Comparison Using Grid Search and Using Fixed $\varepsilon$

\begin{tabular}{|l|c|c|c|c|c|c|c|}
\hline $\mathrm{SNR}(\mathrm{dB})$ & -25 & -24 & -23 & -22 & -21 & -20 \\
\hline $\begin{array}{l}\text { Using grid search to } \\
\text { obtain the highest } \\
\text { probability of } \\
\text { detection }\end{array}$ & $\varepsilon$ & 0.45 & 0.35 & 0.50 & 0.45 & 0.35 & 0.50 \\
\cline { 2 - 8 } & $P_{d}$ & 0.7073 & 0.7545 & 0.8222 & 0.8815 & 0.9345 & 0.9761 \\
\hline $\begin{array}{l}\text { Using fixed } \varepsilon \text { and } \\
\text { double thresholds } \\
\text { detection }\end{array}$ & $\varepsilon$ & 0.25 & 0.25 & 0.25 & 0.25 & 0.25 & 0.25 \\
\cline { 2 - 8 } & $P_{d}$ & 0.7043 & 0.7489 & 0.8002 & 0.8589 & 0.9249 & 0.9681 \\
\hline $\begin{array}{l}\text { Using fixed } \varepsilon \text { and } \\
\text { single threshold } \\
\text { detection }\end{array}$ & $\varepsilon$ & 0.0 & 0.0 & 0.0 & 0.0 & 0.0 & 0.0 \\
\cline { 2 - 8 } & $P_{d}$ & 0.5942 & 0.6513 & 0.7203 & 0.7866 & 0.8643 & 0.9316 \\
\hline
\end{tabular}

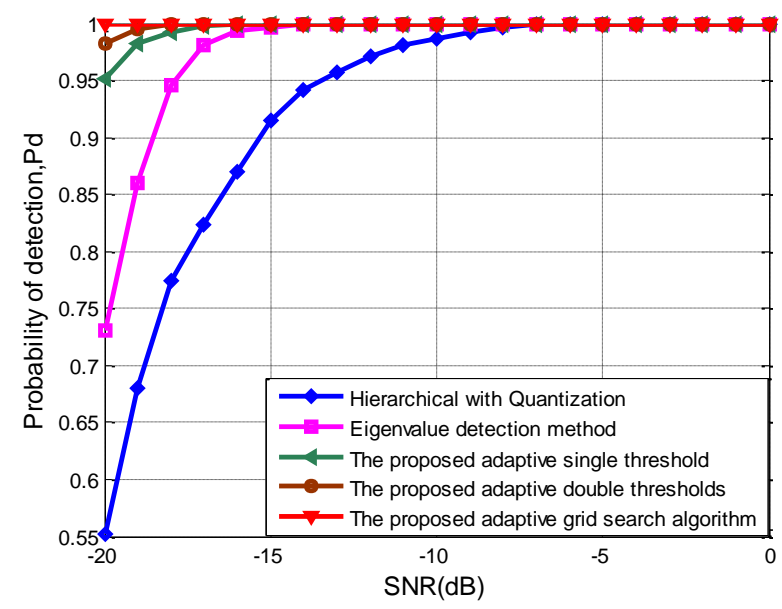

Figure 4. Performance Comparison of the different Sensing Methods 


\section{Conclusion}

In this paper, a novel weighted cooperative spectrum sensing scheme is studied. This scheme used dynamic double energy thresholds to achieve better sensing performance. In local sensing, obtained an optimal energy threshold by minimizing the sum of the probabilities of false alarm and detection, and introduced a control parameter to adjust the local double energy thresholds. In cooperative sensing, each SU was assigned a weight depending on its collected average energy. Moreover, the path loss is taking into consideration in cooperation sensing. Simulation results showed that the proposed spectrum sensing scheme had excellent sensing performance under different SNR.

\section{Acknowledgments}

This work is supported by Education Department of Hunan, China (No. 15C0592); the Construct Program of the Key Discipline in Hunan University of Science and Engineering (Circuits and Systems); the Natural Science Foundation of Zhejiang Province, China (LQ15F010008 and LY15F020040), Science and Technology Program of Jiaxing City, China (2015AY11009).

\section{References}

[1] G. Ganesan and Y. G. Li, "Cooperative spectrum sensing in cognitive radio networks", in Proc. IEEE Symp. New Frontiers in Dynamic Spectrum Access Networks (DySPAN05), Baltimore, USA, pp.137143, (2010), pp. 561--565.

[2] J. Wu, T. Luo and G. Yue, "An energy detection algorithm based on double-threshold in cognitive radio systems," Information Science and Engineering (ICISE), 2009 1st International Conference on. Kyoto, Japan, (1995), IEEE, Kyoto, Japan, (2013), pp. 493-496.

[3] N. Meghanathan, "A Survey on the Communication Protocols and Security in Cognitive Radio Networks", International Journal of Communication Networks and Information Security (IJCNIS), Shanghai, China, (2014), pp.118-124.

[4] S. A. Mousavifar and C. Leung, "Transient Analysis for a Trust-Based Cognitive Radio Collaborative Spectrum Sensing Scheme", IEEE Wireless Communications Letters, vol. 4, (2015), pp. 377-380.

[5] H. Vu-Van and I. Koo, "Cooperative spectrum sensing with double adaptive energy thresholds and relaying users in cognitive radio", Telecommunications (AICT), 2010 Sixth Advanced International Conference on. IEEE, Xian, China, (2013), pp. 52-56.

[6] Z. Shibing, Y. Jiaojiao and G. Lili, "Eigenvalue-Based Cooperative Spectrum Sensing Algorithm", in Instrumentation, Measurement, Computer, Communication and Control (IMCCC), 2012 Second International Conference on, Hong Kong, China, (2012), pp. 375-378.

[7] A. Bagwari and G. S. Tomar, "Adaptive double-threshold based energy detector for spectrum sensing in cognitive radio networks", International Journal of Electronics Letters, (2013), pp. 33-41.

[8] T. Kieu-Xuan and I. Koo, "A cooperative spectrum sensing scheme using adaptive fuzzy system for cognitive radio networks", Information Sciences, vol. 220, (2013), pp. 102-109.

[9] L. Xiang, W. Bin, W. Hong, H. Pin-Han, B. Zhiqiang and P. Lili, "Adaptive Threshold Control for Energy Detection Based Spectrum Sensing in Cognitive Radios", Wireless Communications Letters, IEEE, vol. 1, (2012), pp. 448-451.

[10] N. Zhao, "A novel two-stage entropy-based robust cooperative spectrum sensing scheme with two-bit decision in cognitive radio", Wireless Personal Communications, (2013), pp. 1-15.

[11] S. Zhang, X. Dong, Z. Bao and H. Zhang, "Adaptive Spectrum Sensing Algorithm in Cognitive UltraWideband Systems", Wireless Personal Communications, vol. 68, (2013), pp. 789-810.

[12] A. Mohammadi, M. R. Taban, J. Abouei and H. Torabi, "Fuzzy likelihood ratio test for cooperative spectrum sensing in cognitive radio", Signal Processing, vol. 56, (2014), pp. 62-66.

[13] I. Sobron, P. S. R. Diniz, W. A. Martins and M. Velez, "Energy Detection Technique for Adaptive Spectrum Sensing", IEEE TRANSACTIONS ON COMMUNICATIONS, vol. 63, (2015), pp. 617-627.

[14] A. Salman, I. M. Qureshi, K. Sultan and S. Saeed, "Joint spectrum sensing for detection of primary users using cognitive relays with evolutionary computing," Communications, IET, vol. 9, (2015), pp. 1643-1648.

[15] D. Guoru, W. Qihui, S. Fei, and W. Jinlong, "Decentralized sensor selection for cooperative spectrum sensing based on unsupervised learning", in Communications (ICC), 2012 IEEE International Conference on, Gaungzhou, China, (2015), pp. 1576-1580. 


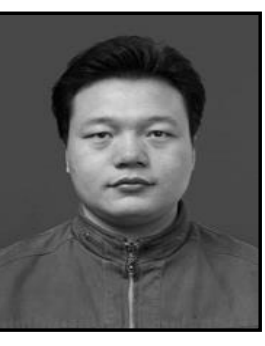

\section{Authors}

Tangsen Huang, he received B.Eng. degree in Electronic Information Engineering from Hunan University of Technology, Zhuzhou, China, in 2004. He received M. Eng. Degree in Signal and Information Processing from Guilin University of Electronic Science and Technology, Guilin, China, in 2007. Since September 2007, he worked as a teacher in Hunan University of Science and Engineering, Yongzhou, China. He is pursuing Ph.D. in the School of Electronic and Information Engineering, South China University of Technology, Guangzhou, China. His research interests are wireless communication, cognitive radio, ultra-wideband and wireless sensor networks.

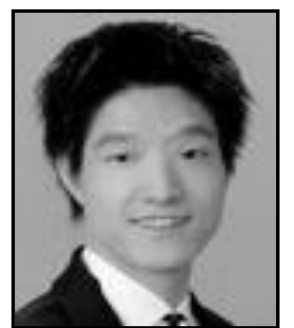

Jie-tai Wang, he received the bachelor's degree in electronic and information engineering from Gannan Normal University, Ganzhou, China, in 2002. He received the Master's Degree and Ph.D in Communication and Information System from Northwestern Polytechnical University, Xi'an, China, in 2005 and 2009 respectively. Since 2012, he has been a teacher in Jiaxing University. His current interest is wireless communication. 
International Journal of Future Generation Communication and Networking Vol. 9, No. 12 (2016) 